\title{
A Dendrite-free non-Newtonian Semisolid Lithium Metal Anode
}

Yunbo Zhang, ${ }^{1,+}$ Zhiyuan Han, ${ }^{1,+}$ Zhijia Huang, ${ }^{1}$ Chen Zhang, ${ }^{1}$ Chong Luo, ${ }^{1}$ Guangmin Zhou, ${ }^{1}$ Wei

$$
\text { LV, }{ }^{1, *} \text { \& Quan-Hong Yang } 2, *
$$

${ }^{1}$ Shenzhen Geim Graphene Center, Tsinghua-Berkeley Shenzhen Institute \& Tsinghua Shenzhen International Graduate School, Tsinghua University, Shenzhen 518055, China.

${ }^{2}$ Nanoyang Group, State Key Laboratory of Chemical Engineering, School of Chemical Engineering and Technology, Tianjin University, Tianjin 300072, China

$\dagger$ These authors are equal main contributors to this work

\section{AUTHOR INFORMATION}

* Correspondence should be addressed to W. L. (lv.wei@sz.tsinghua.edu.cn) and Q.-H. Y. (qhyangcn@tju.edu.cn) 


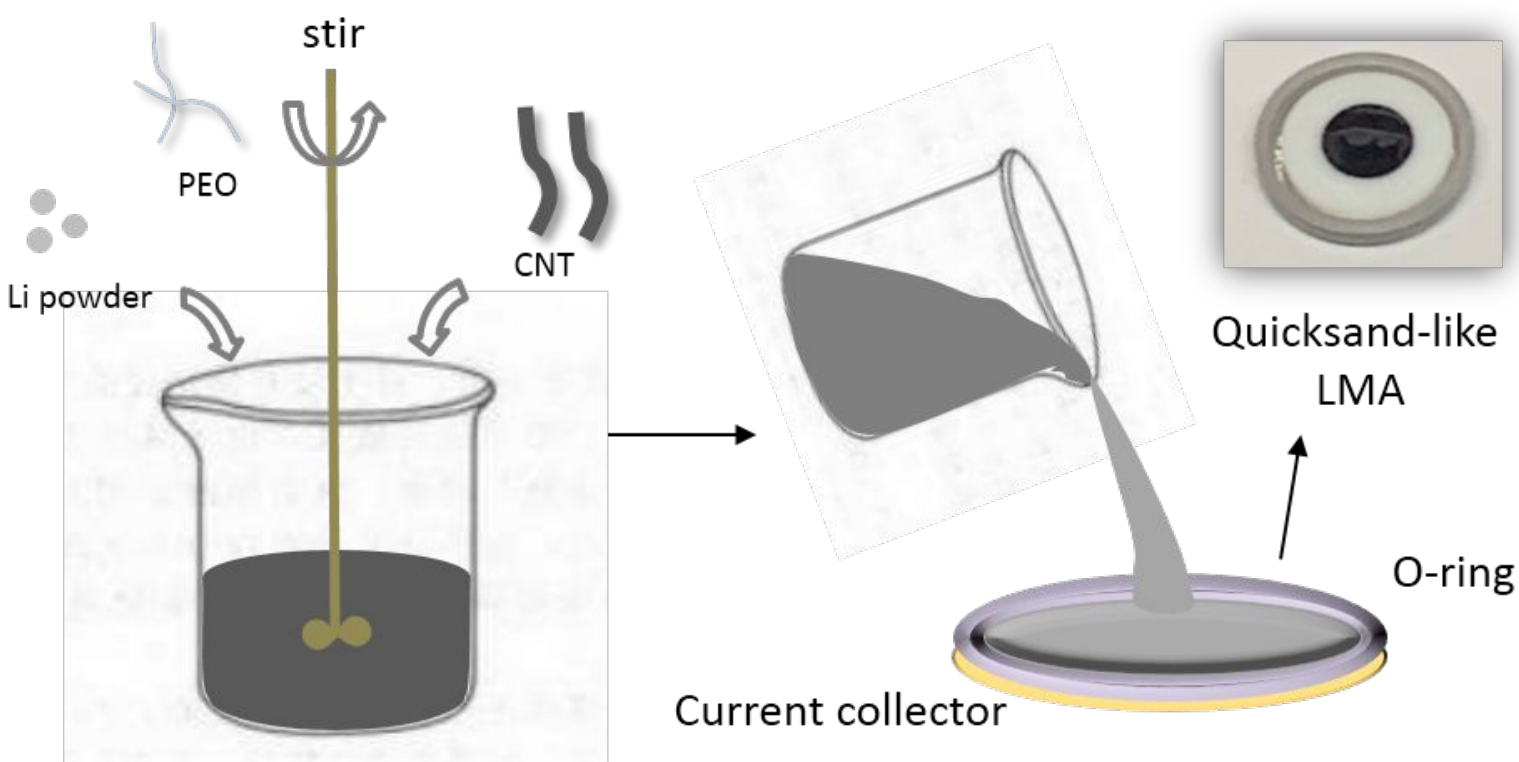

Figure S1. The preparation process of the quicksand-like LMA.
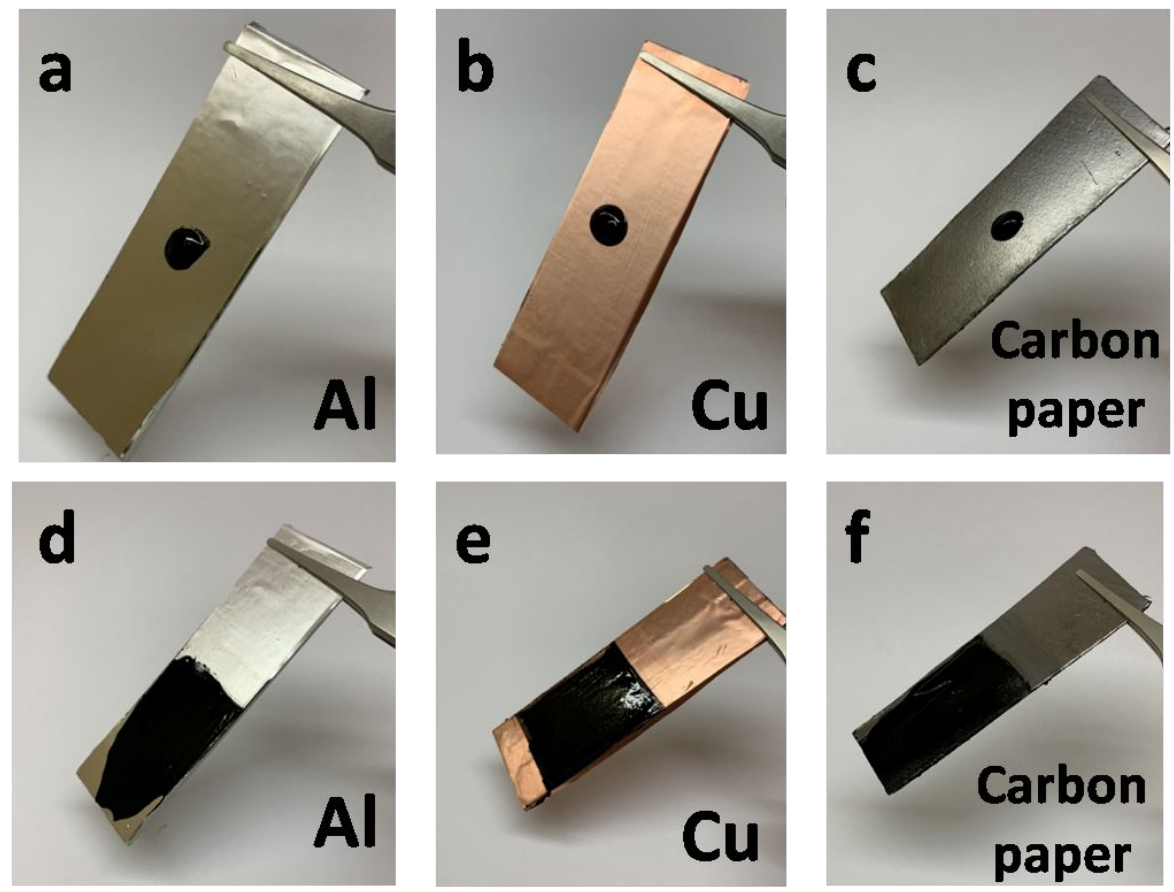
Figure S2. The photos of quicksand-like LMA drops on tilted (a) aluminum foil, (b) copper foil and (c) carbon paper. The photos of the quicksand-like LMA coated on the tilted (d) aluminum foil, (e) copper foil and (f) carbon papers.

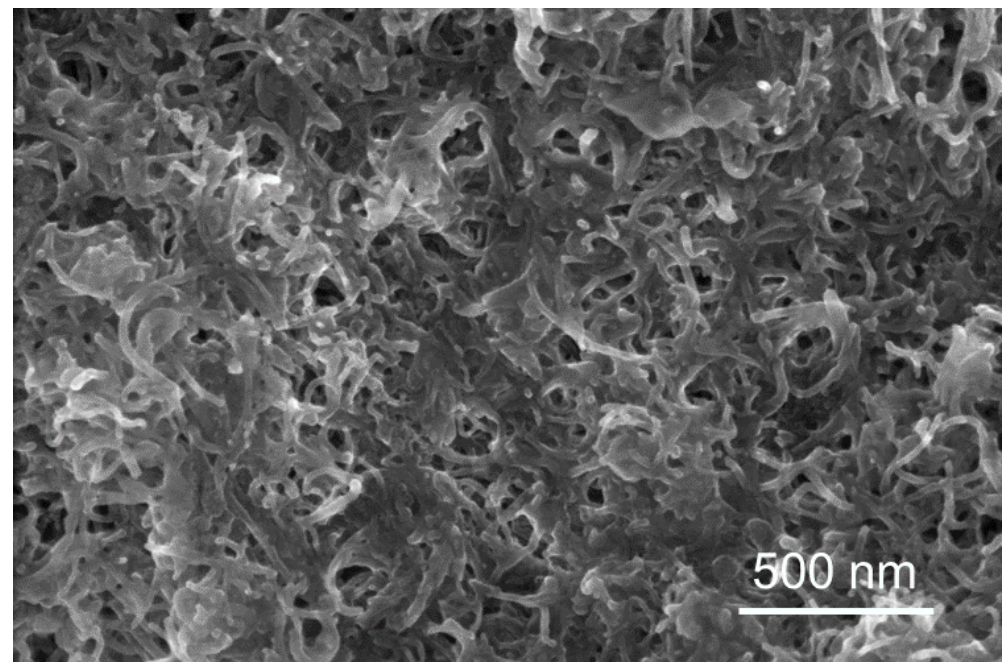

Figure S3. The high- magnification SEM image of the quicksand-like fluid after the liquid evaporated.
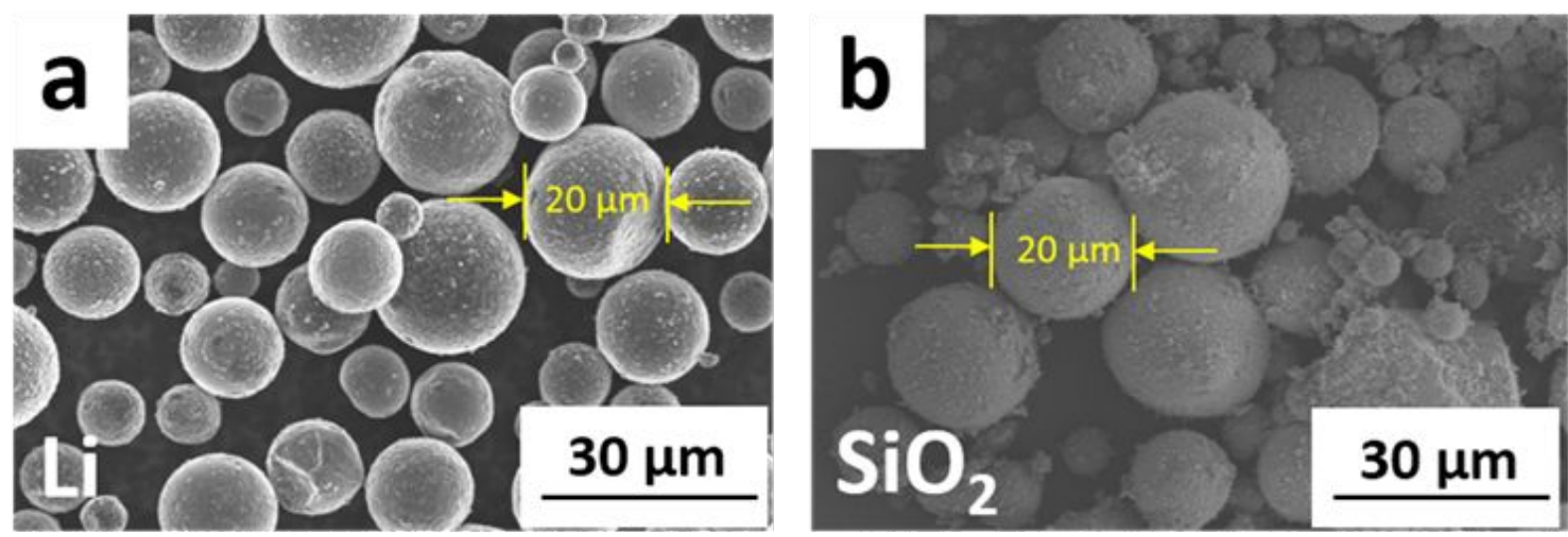

Figure S4. The SEM images of (a) the lithium powders and (b) silica powders 

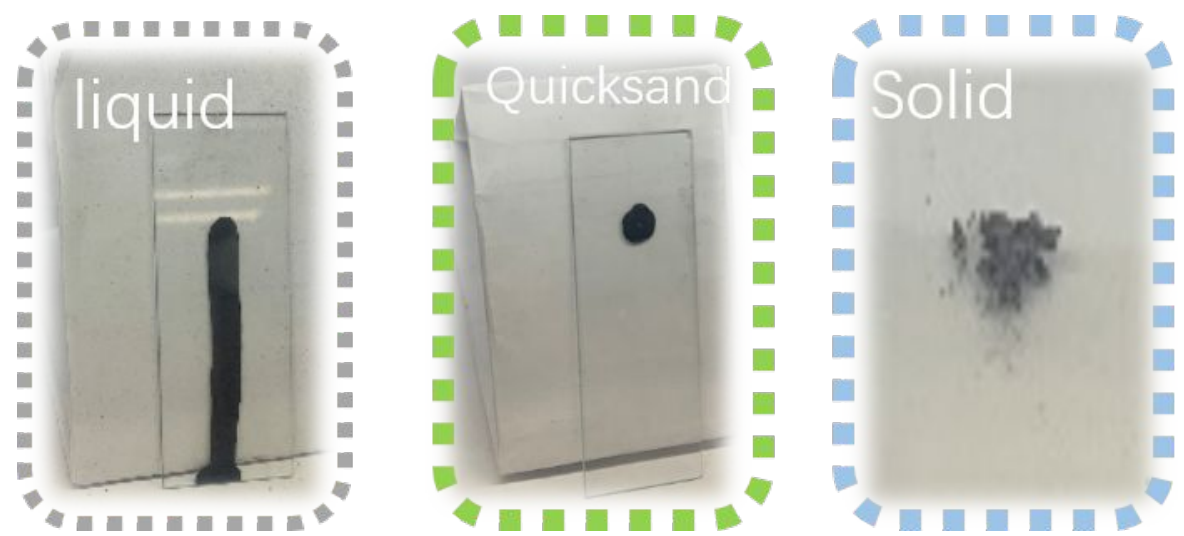

Figure S5. The photos of the three phases of the samples.

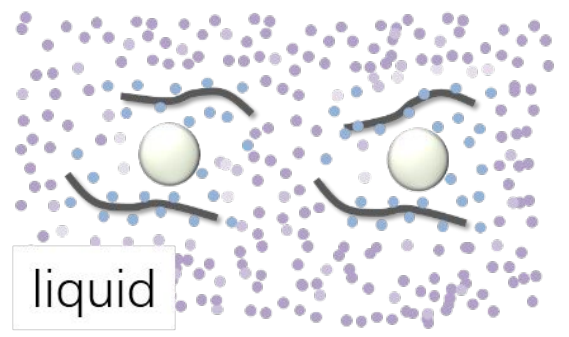

Free liquid

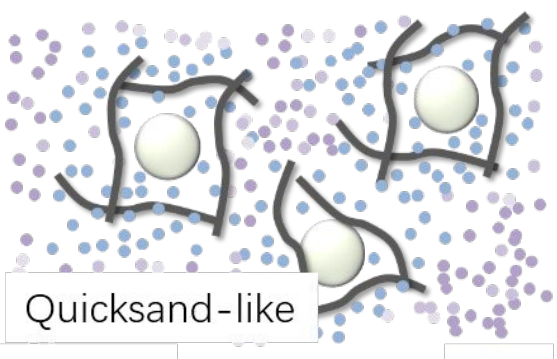

Adsorbed liquid

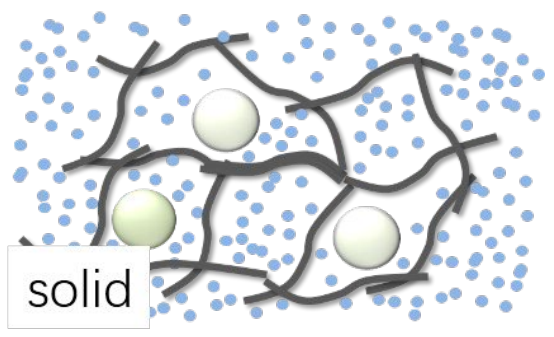

Li powder

Figure S6. The diagrams of three phases.
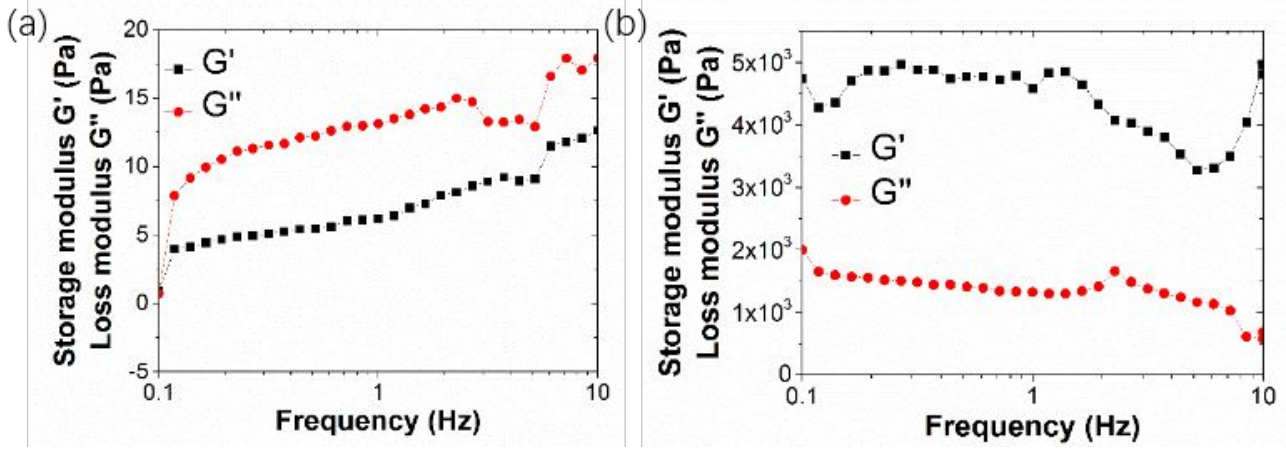

Figure S7. The rheology analysis by DMA of the samples of (a) liquid phase and (b) solid phase. 

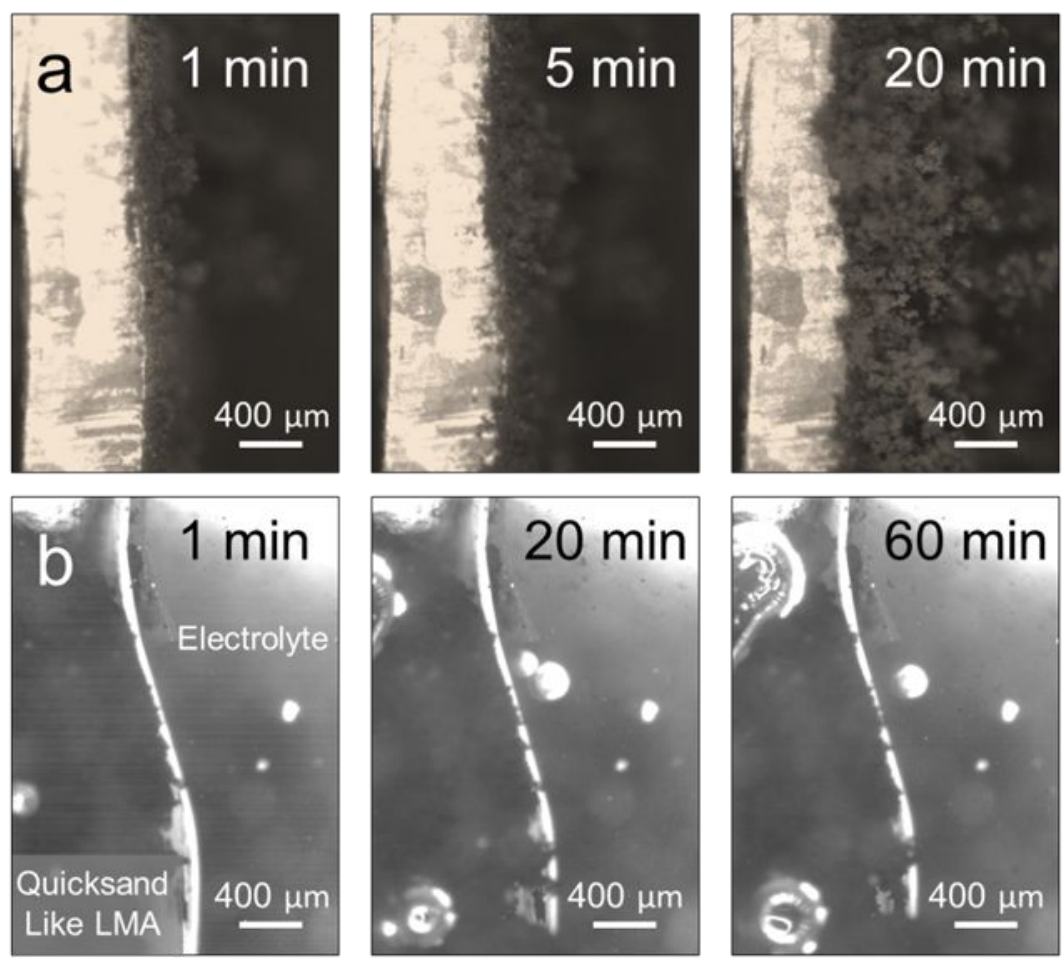

Figure S8. The in situ optical microscope images showing the plating process of (a) the Li metal and (b) quicksand-like LMA in the electrolyte without $\mathrm{LiNO}_{3}$ at the current density of $3 \mathrm{~mA} \mathrm{~cm}$.

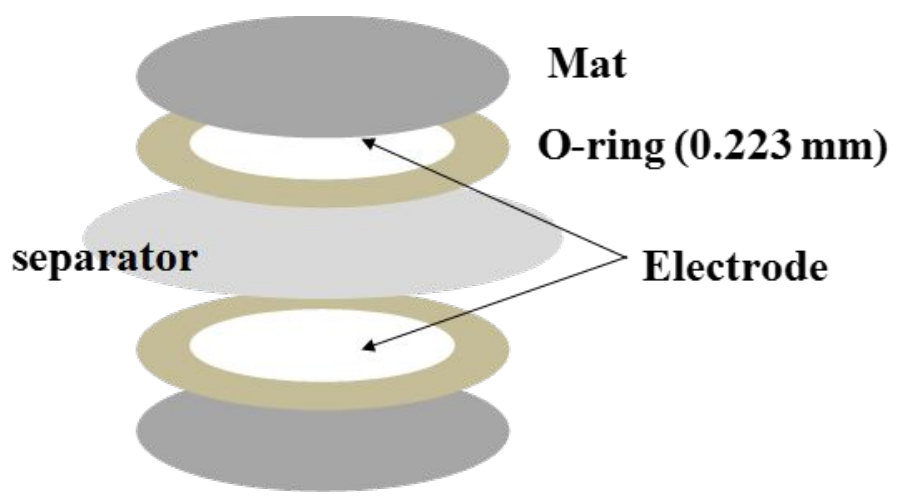

Figure S9. The assembling method of the quicksand-like LMA. 


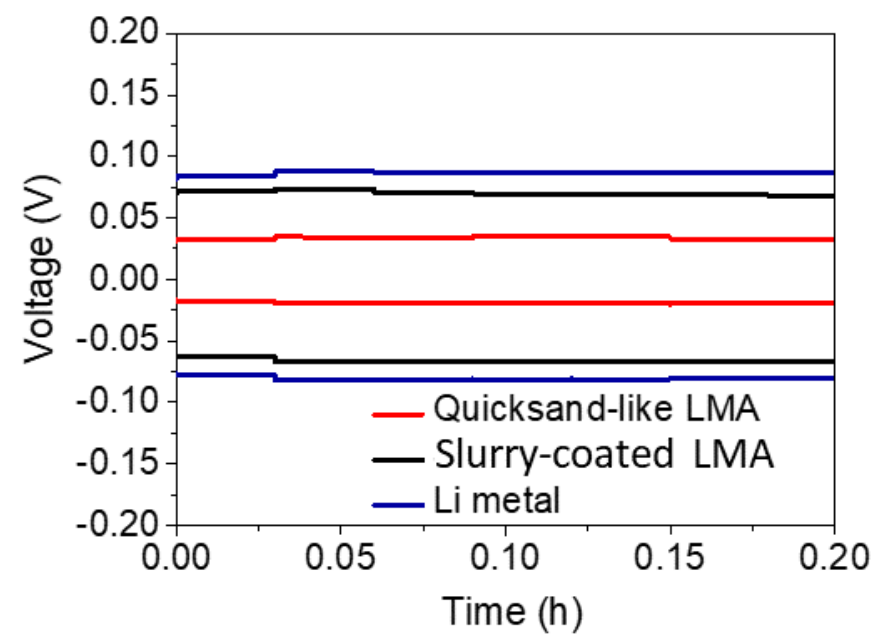

Figure S10. The zoom picture of the plating/stripping curve in Figure 2c.
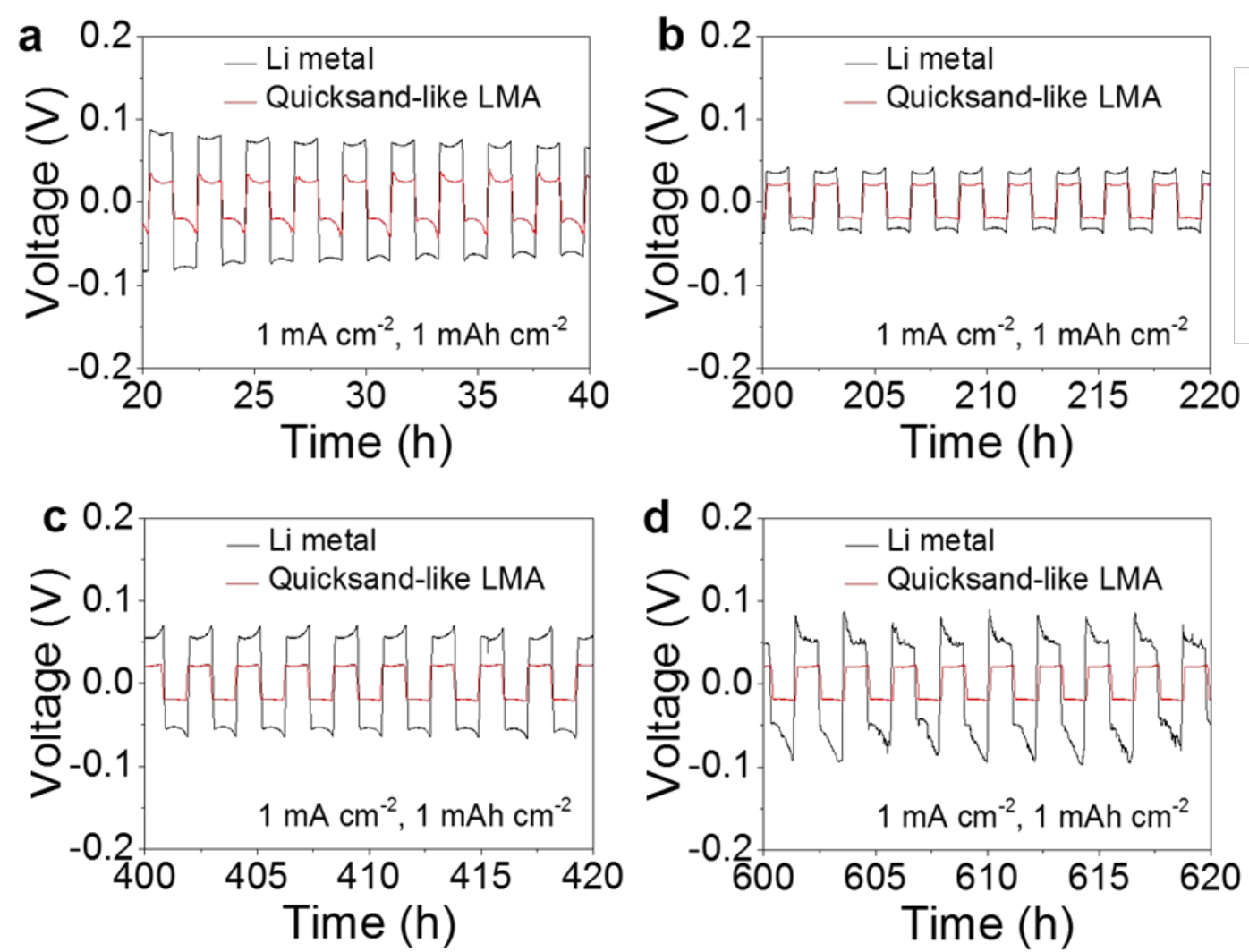

Figure S11. The overpotential at different cycling time of Li metal and quicksand-like LMA at $1 \mathrm{~mA} \mathrm{~cm}^{-2}$ and $1 \mathrm{mAh} \mathrm{cm}^{-2}$. 

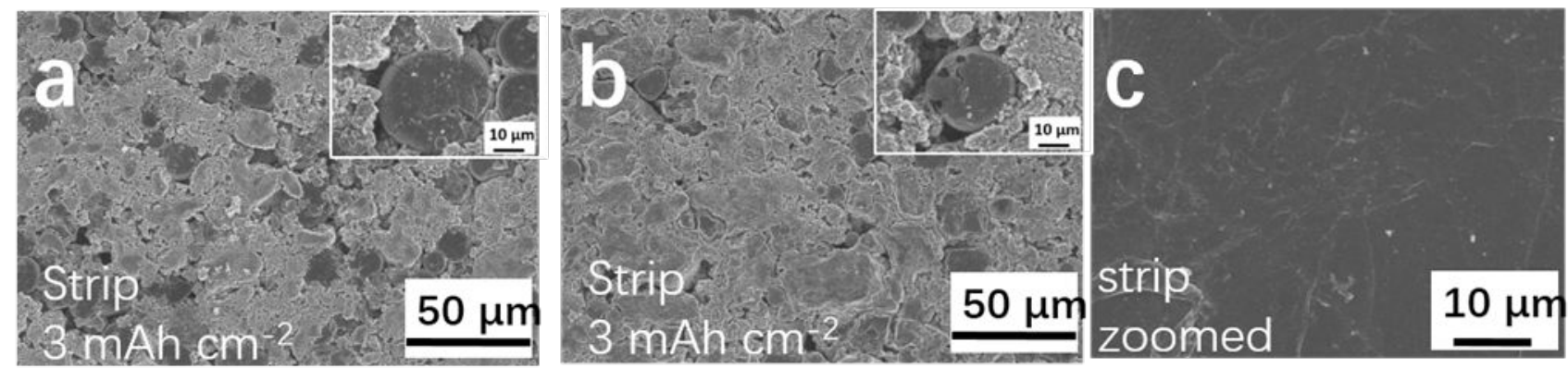

Figure S12. The SEM images of the surfaces of (a) quicksand-like LMA, (b) slurry coated LMA and (c) Li metal after plating processes (the inserts are the zoomed images). The current density applied was 1 $\mathrm{mA} \mathrm{cm} \mathrm{cm}^{-2}$, and the stripping capacity was $3 \mathrm{mAh} \mathrm{cm}^{-2}$.

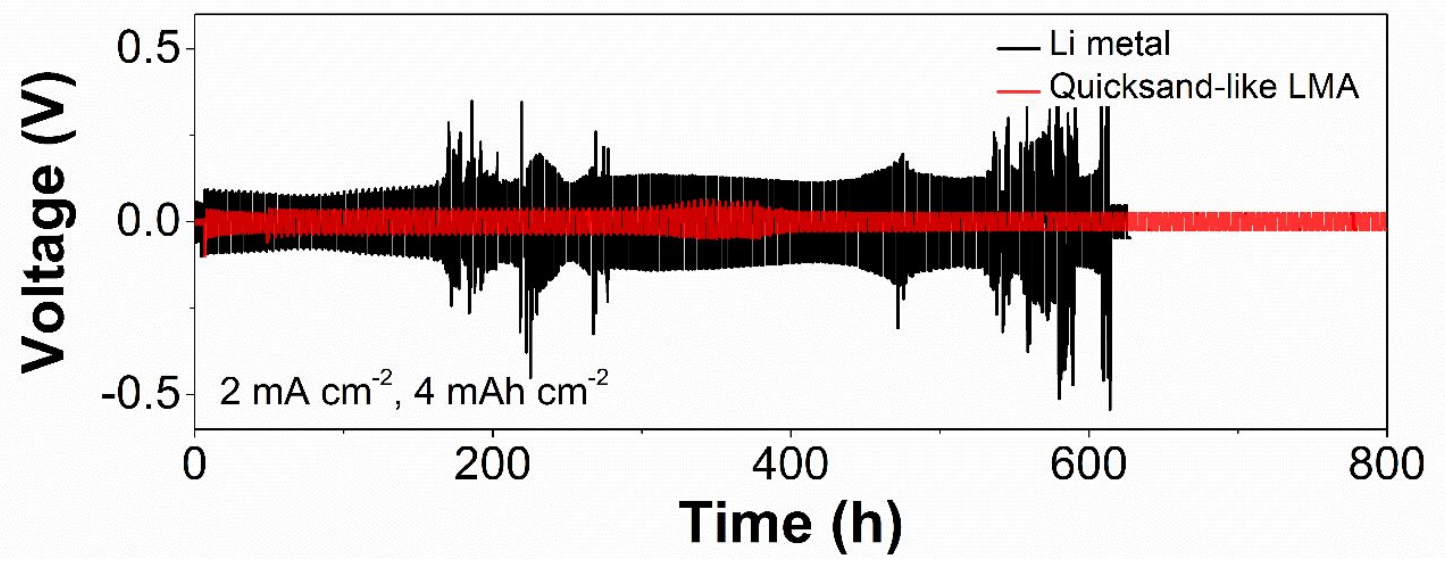

Figure S13. The cycling performance of the quicksand-like LMA and Li metal at the rate of $2 \mathrm{~mA} \mathrm{~cm}{ }^{-2}$ with the capacity of $4 \mathrm{mAh} \mathrm{cm}^{-2}$. 

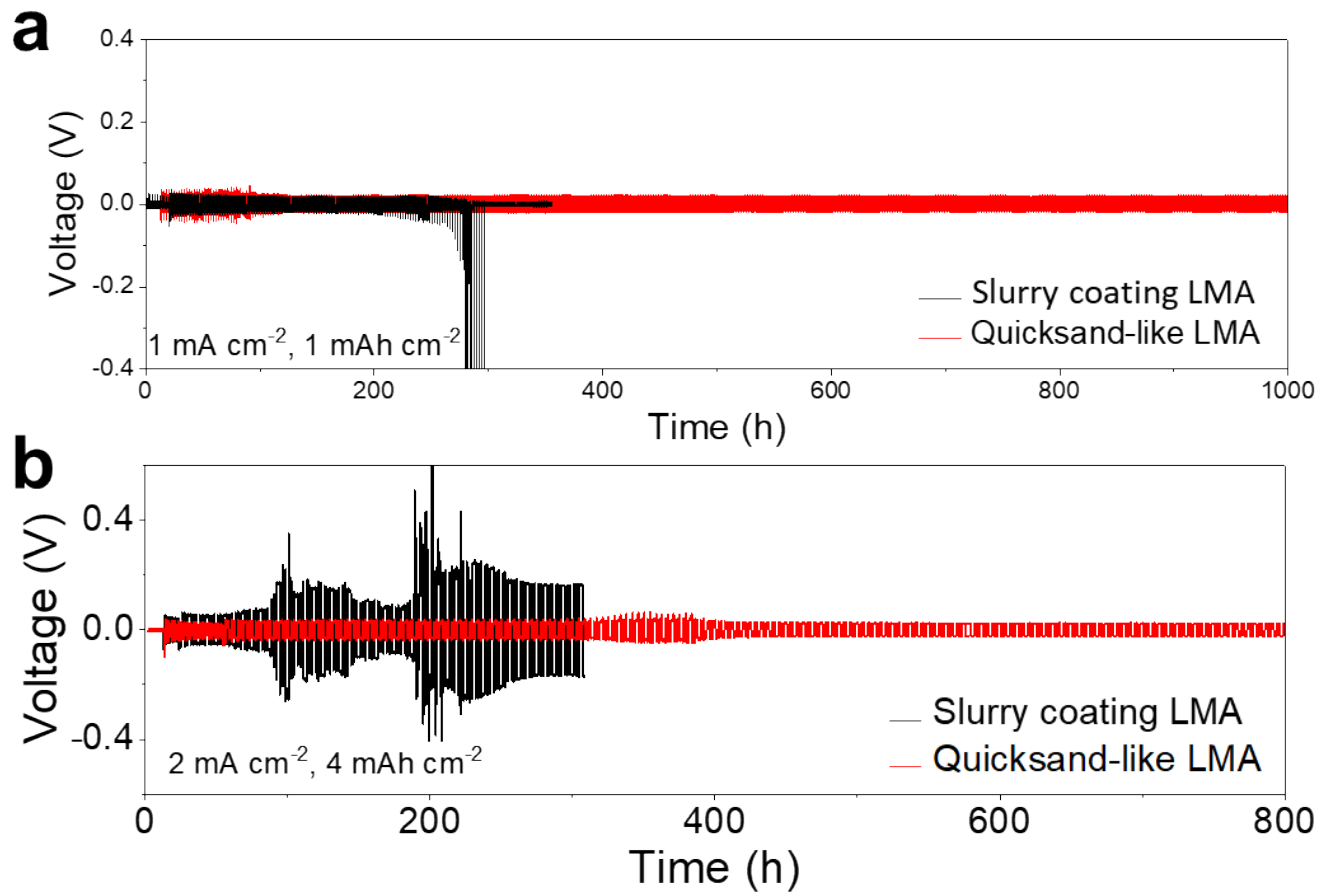

Figure S14. The cycling performance of the quicksand-like LMA and slurry coating LMA (a) at the rate of $1 \mathrm{~mA} \mathrm{~cm}^{-2}$ with the capacity of $1 \mathrm{mAh} \mathrm{cm}^{-2}$ and (b) at the rate of $2 \mathrm{~mA} \mathrm{~cm}{ }^{-2}$ with the capacity of $4 \mathrm{mAh}$ $\mathrm{cm}^{-2}$.
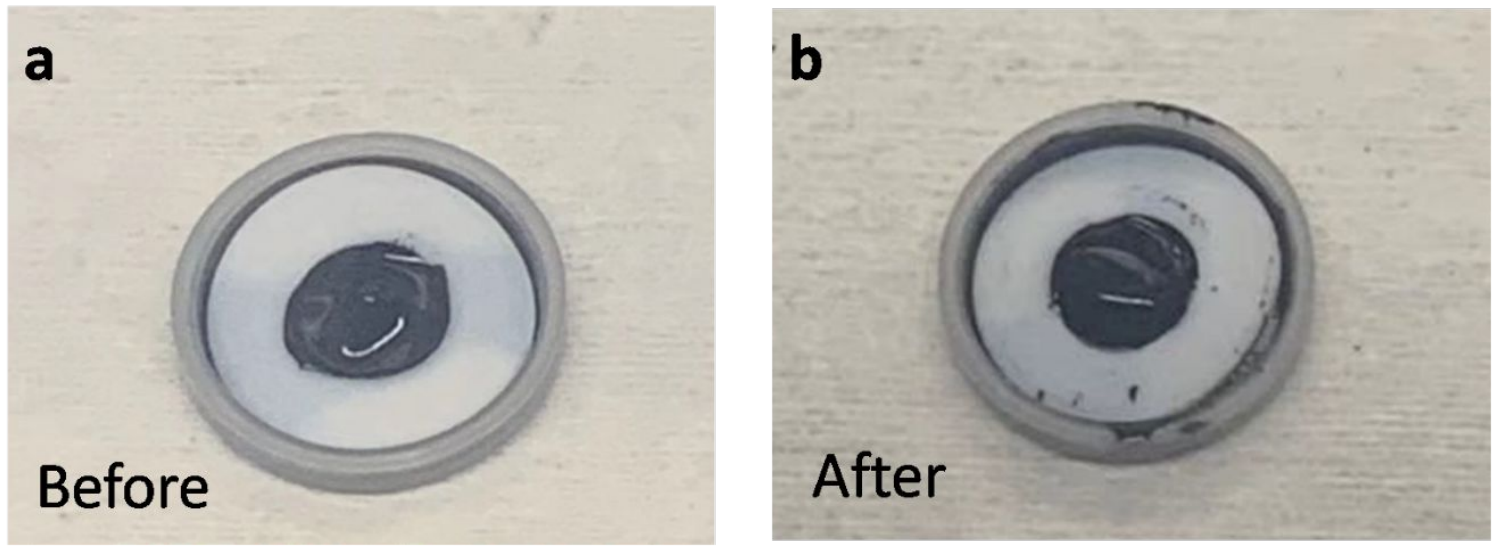

Figure S15. The macroscale photos of quicksand-like LMA in coin cells (a) before and (b) after 50 cycles. 

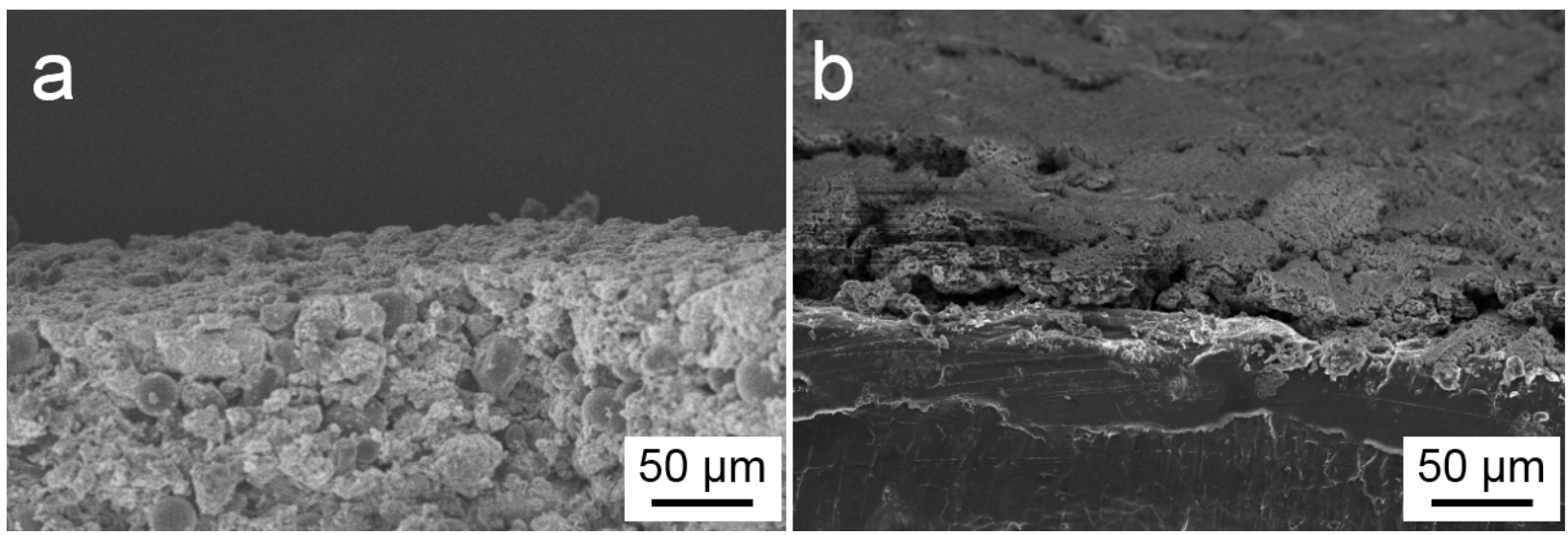

Figure S16. The cross-sectional SEM images of (a) quicksand-like LMA and (b) Li metal anode after 50 cycles.
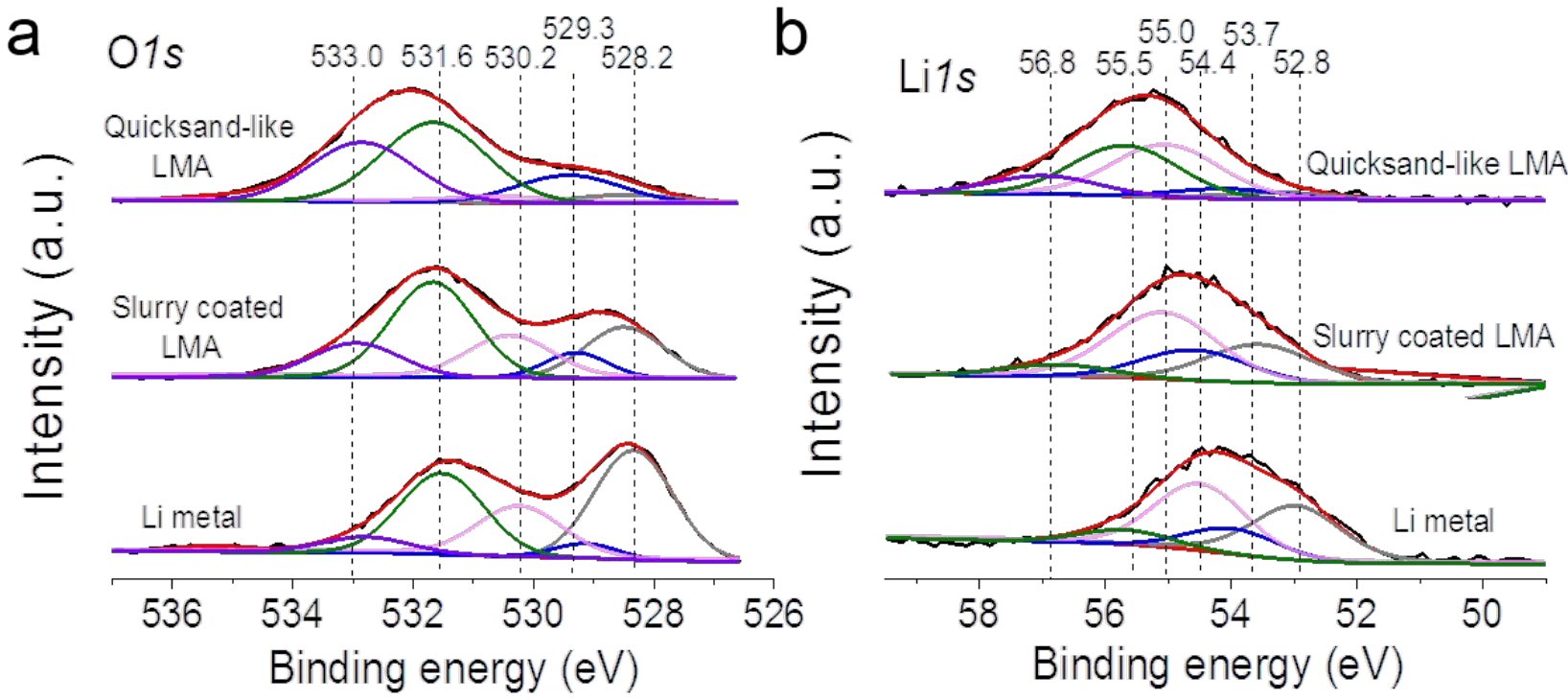

Figure S17. The XPS data of the Li metal, the slurry coating LMA and the quicksand-like LMA after 20 cycles at the rate of $1 \mathrm{~mA} \mathrm{~cm}{ }^{-2}$ with the capacity of $1 \mathrm{mAh} \mathrm{cm}^{-2}$. (a) The $\mathrm{O} 1 \mathrm{~s}$ spectra and (b) the Li $1 s$ spectra. 

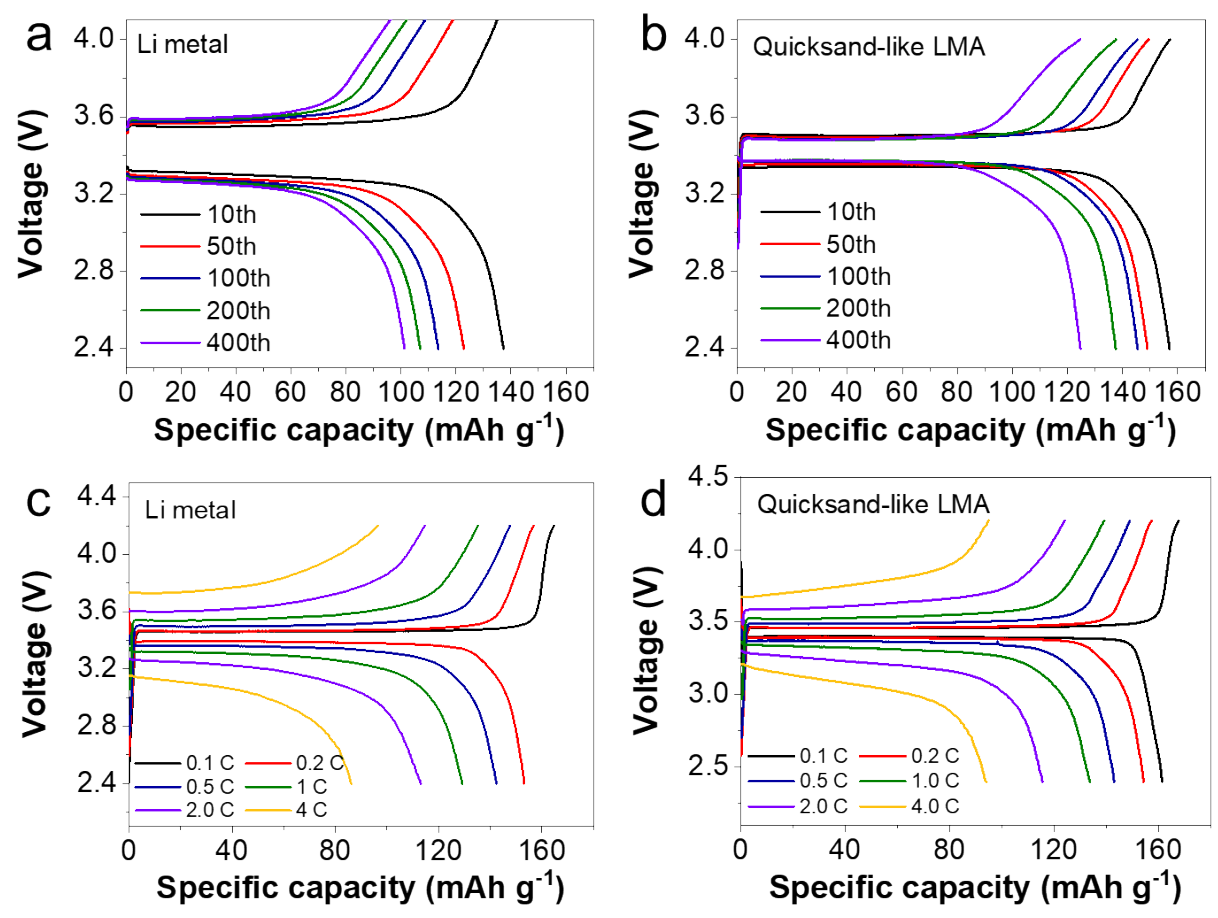

Figure S18. The charging and discharging profiles of the cells with Li metal anode (a and c) and quicksand-like LMA (b and d) at different cycles and rates.

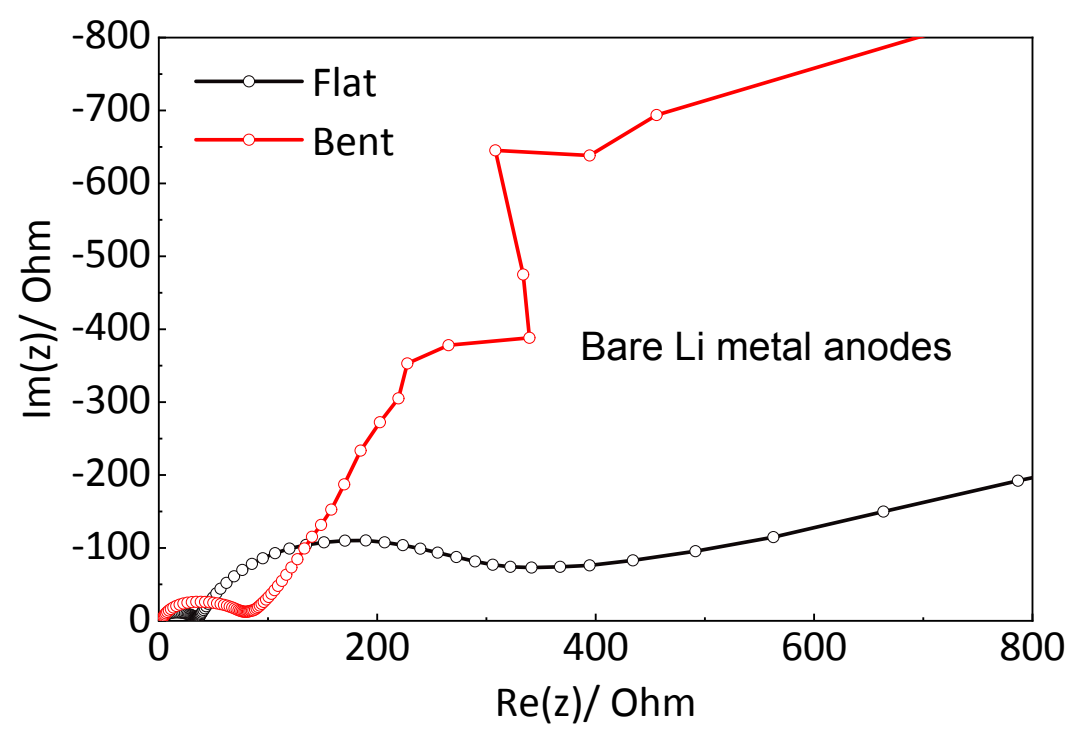

Figure S19. The EIS of the cells with the bare Li before and after bending. 